\title{
Is there a risk of chikungunya transmission in Canada?
}

\author{
Ogden $\mathrm{NH}^{1^{*}}$, Lindsay $\mathrm{LR}^{2}$, Coulthart $\mathrm{M}^{1}$ \\ ${ }^{1}$ Centre for Food-borne, Environmental and Zoonotic Infectious Diseases, Public Health Agency of Canada, Ottawa, ON \\ ${ }^{2}$ Zoonotic Diseases and Special Pathogens, Public Health Agency of Canada, Winnipeg, MB
}

${ }^{*}$ Corresponding author: Nicholas.ogden@phac-aspc.gc.ca

\section{Abstract}

An outbreak of chikungunya virus infection is currently underway in the Caribbean, Central America and South America, and autochthonous (local or indigenous) transmission has occurred in the southeastern United States. The mosquito species known to transmit chikungunya-Aedes aegypti and Aedes albopictus-are not known to reside in Canada at this time. But how comfortable can we be that this situation will continue? Here we explore four key conditions that must be met for transmission of chikungunya within Canada. We conclude that not all of these conditions have been met and the risk of chikungunya transmission in most of Canada appears to be very low at present. The risk is slightly higher in warmer areas, such as southern British Columbia and isolated locations in south central and southeastern Canada. However, there are significant gaps in our knowledge and ongoing risk assessment, research and surveillance for vectors of chikungunya are indicated.

\section{Introduction}

The mosquito-borne viral illness chikungunya is endemic to parts of Africa, Asia, and the Indian and Pacific Oceans. In December 2013, two autochthonously (locally or indigenously) transmitted cases of chikungunya on the Caribbean island of Saint-Martin/Sint Maarten marked the beginning of an outbreak in the western hemisphere. Over the course of 2014, chikungunya spread through much of the Caribbean, as well as parts of Central America, South America and Mexico, with autochthonous transmission being seen in the southeastern United States $(1,2)$.

A rapid increase in laboratory diagnoses of travel-acquired chikungunya infections in Canadians has been seen recently (3). This would be expected, given the high numbers of Canadians who travel to affected regions for business and pleasure (3). In addition to the direct risk of infections being acquired during travel, what is the risk that travellers who have acquired infection abroad return and act as a source of local autochthonous chikungunya transmission in Canada? In this article, we evaluate the possibility of local transmission of chikungunya virus in Canada that could lead to isolated autochthonous cases or outbreaks.

\section{Analysis}

There are four conditions that must be met for local transmission of chikungunya to occur: the introduction of the virus; the presence of a competent vector; suitable climactic conditions for virus transmission; and a sufficient number of susceptible people. We examine each of these conditions below and identify whether or not these conditions are currently present in Canada.

\section{Introduction of chikungunya virus}

Travellers, including Canadians, who acquire chikungunya are frequently viraemic on their return and could be the source for infection of Canadian residents if competent vector mosquitoes were present and other conditions of transmission were met (3). Infected, viraemic returning travellers are thought to be the most important source of international spread of chikungunya virus and the most likely source of introduction of infection for outbreaks in Italy, as well as autochthonous cases in France and the United States $(4,5,6)$. Introduction of infected mosquitoes could occur with international trade through products that carry live infected mosquitoes from affected 
regions (4). Studies are underway to identify potential hotspots in Canada for introduction of chikungunya virus in people or mosquito-contaminated imports.

\section{Presence of a competent vector}

For transmission to occur in Canada from an infected returning traveller to a susceptible Canadian resident, mosquitoes of a species capable of transmitting the virus must be present. The only two vectors known to be capable of transmitting chikungunya virus, and to have played a role in a chikungunya outbreak, are the mosquitoes Aedes aegypti (the yellow fever mosquito) and Aedes albopictus (the Asian tiger mosquito) $(2,4)$. The main determinant of where these mosquitoes can become established is climate. Both species are implicated in the transmission of chikungunya in the current outbreak in the Americas (1).

Aedes aegypti is a mosquito adapted to sub-tropical and tropical regions and is unlikely to become established in Canada under our current climate conditions or even with climate change (7). Aedes albopictus, however, can survive cooler northern temperatures. It is able to survive cold winters, and became established in parts of the United States in the 1980s (8). This mosquito now occurs in central and eastern regions of the U.S., and extending to the southern parts of states that border Canada, including New York, Pennsylvania and Ohio. It has also occurred in, and been eradicated from, several western states (8). However, our current knowledge of where Aedes albopictus is in North America is based primarily on informal surveillance and field studies. Surveillance that would identify whether and where this mosquito is becoming established in risk areas in Canada is being planned with provincial partners.

Recent studies suggest that the climate in most of Canada (except for southern coastal British Columbia and some locations in south central and southeastern Canada) is not suitable for long-term survival of the Aedes albopictus mosquito (9). There are rare reports of this mosquito in Canada (10), but there is no evidence that it has become established in any part of Canada. This situation may change with a changing climate. Assessing how much change would be needed for Aedes albopictus mosquitoes to become established needs more research (9).

\section{Presence of suitable climatic conditions}

There are two climatic factors that determine whether conditions are suitable for chikungunya transmission. First, the climate has to be suitable to support not only reproducing populations of the mosquito species, but populations with an abundance high enough such that at least one mosquito acquires chikungunya virus from an infected person and survives to transmit it to an uninfected person. Second, the temperature conditions have to be warm enough for chikungunya virus to spread from the mosquito's gut (where the virus is first present after being acquired from an infected person) to the salivary glands (from which the virus is transmitted in saliva when the mosquito next feeds) before the mosquito dies. Recent studies suggest a threshold temperature of $20^{\circ} \mathrm{C}$ for the occurrence of outbreaks of chikungunya based on past experiences (10). We are currently investigating where and when climatic conditions are suitable for chikungunya transmission in Canada under current and future climate. Outputs of this research will be combined with assessments of where risk mosquito vectors may occur to improve our assessment of risk of autochthonous transmission and to guide public health programs.

\section{Presence of sufficient numbers of susceptible people}

If there were locations in Canada where competent mosquitoes were established in sufficient numbers for transmission and were exposed to infected persons returning from affected countries, individual autochthonous cases of chikungunya could occur. However, for transmission to be sustained in the short or long term, infected mosquitoes would need to have access to a sufficient number of susceptible people.

In developing countries, mosquitoes have relatively free access to both buildings and the people that occupy them. In countries such as Canada, however, residences and businesses are mostly well-sealed off from the incursion of mosquitoes, and mosquito bites mostly occur when people are outside. Typically, this does not involve large crowds of people, but rather hikers, gardeners, and others in relatively low density. In the absence of sufficient numbers of humans, infected Aedes albopictus mosquitoes may be more likely to feed on wild or domesticated animals, thus inhibiting transmission in humans. These socioeconomic factors are thought to limit the transmission of other vector-borne diseases, such as dengue, and will likely be a key factor in dampening any putative autochthonous transmission of chikungunya in countries such as Canada (11). 


\section{Discussion and conclusion}

In light of the current outbreak of chikungunya occurring south of us in the Americas, assessing the risks of autochthonous cases or limited outbreaks of chikungunya in Canada is indicated. At present, there is no evidence that competent vectors of chikungunya, such as Aedes albopictus, are established in Canada, and climatic conditions appear currently mostly unsuitable or sub-optimal for this species. Therefore, presently, the risk of autochthonous cases and outbreaks of chikungunya in Canada appears to be very low. However, the risk is slightly higher in warmer climate areas, such as southern British Columbia and isolated locations in south central and southeastern Canada. Furthermore, socioeconomic factors may also be unfavourable for human-to-mosquitoto-human transmission of the virus.

The primary risk of autochthonous transmission in Canada would arise from Aedes albopictus mosquitoes becoming established here. Our knowledge of its current whereabouts is based on informal surveillance. More field research is needed in North America to better understand and predict the climatic limits of this species (10).

Several factors could alter this assessment. Temperatures suitable for chikungunya virus transmission are achieved at times in many parts of Canada during the summer (9), and it is possible that in some circumstances housing conditions and densities of uninfected people would be sufficient to sustain limited outbreaks.

Furthermore, it is possible that genetic plasticity of mosquito species could improve their capacity to survive in cooler, more northerly climates, although this is difficult to predict (10). Finally, viral mutations could theoretically result in viruses becoming transmissible by other vectors that are established in Canada (2).

In conclusion, our observations suggest that the current risk of autochthonous spread of chikungunya virus in Canada is very low, but there are gaps in our knowledge and several factors could alter this risk assessment. This suggests the need for ongoing risk assessment, consideration of the projected effects of climate change, surveillance for human cases of chikungunya, enhanced surveillance for mosquito vectors, and the development of plans for prevention and control of outbreaks of this and other exotic vector-borne diseases that may threaten the health of Canadians in the coming decades.

\section{Acknowledgements}

None

\section{Conflict of interest}

None

\section{Funding}

None

\section{References}

(1) Pan American Health Organization. Number of reported cases of chikungunya fever in the Americas-EW 40 (October 24, 2014). http://www.paho.org/hq/index.php?option=com_topics\&view=article\&id=343\&ltemid=40931

(2) Powers AM. Risks to the Americas associated with the continued expansion of Chikungunya virus. J Gen Virol. 2014 pii: vir.0.070136-0.

(3) Drebot, MA, Holloway K, Zheng H, Ogden NH. Travel-related Chikungunya cases in Canada, 2014. CCDR. 2015 Jan 8;41(1):2-6.http://www.phac-aspc.gc.ca/publicat/ccdr-rmtc/14vol41/index-eng.php

(4) Charrel RN, De Lamballerie X, Raoult D. Chikungunya outbreaks-The globalization of vectorborne diseases. N Engl J Med. 2007;356:769-71.

(5) La Ruche G, Souarès Y, Armengaud A, Peloux-Petiot F, Delaunay P, Desprès P, et al. First two autochthonous dengue virus infections in metropolitan France. Euro Surveill. 2010;15:19676.

(6) Johansson MA, Powers AM, Pesik N, Cohen NJ, Staples JE. Nowcasting the spread of chikungunya virus in the Americas. PLoS One. 2014;9:e104915.

(7) Khormi HM, Kumar L. Climate change and the potential global distribution of Aedes aegypti: Spatial modelling using GIS and CLIMEX. Geospat Health. 2014;8:405-15. 
(8) Moore CG. Aedes albopictus in the United States: Current status and prospects for further spread. J Am Mosq Control Assoc. 1999;15:221-7.

(9) Fischer D, Thomas SM, Suk JE, Sudre B, Hess A, Tjaden NB, et al. Climate change effects on Chikungunya transmission in Europe: Geospatial analysis of vector's climatic suitability and virus' temperature requirements. Int $\mathrm{J}$ Health Geogr. 2013;12:51.

(10) Ogden NH, Radojevic M, Caminade C, Gachon P. Recent and projected future climatic suitability of North America for the Asian tiger mosquito Aedes albopictus. Parasites \& Vectors. 2014 Dec 2;7:532.

(11) Reiter P. Climate change and mosquito-borne disease. Environ Health Perspect. 2001;109(Suppl 1):141-61. 